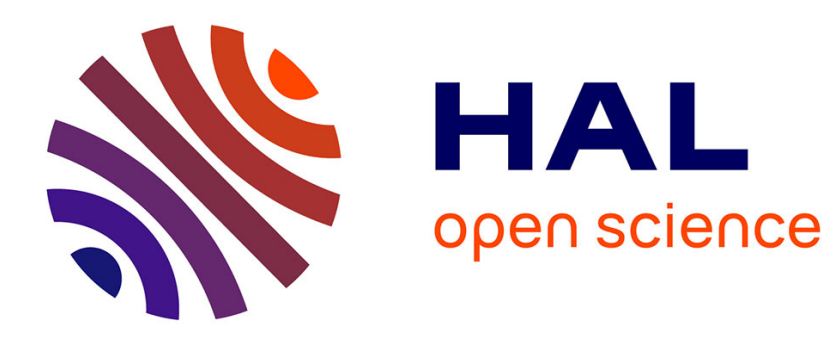

\title{
Performance et vieillissement sportif
}

\author{
Geoffroy C.B. Berthelot
}

\section{To cite this version:}

Geoffroy C.B. Berthelot. Performance et vieillissement sportif . Gérontologie et Société, 2015, Regards croisés sur le corps vieillissant, 37 (148), pp.133-142. 10.3917/gs1.148.0135 . hal-01769447

\section{HAL Id: hal-01769447 https://hal-insep.archives-ouvertes.fr/hal-01769447}

Submitted on 18 Apr 2018

HAL is a multi-disciplinary open access archive for the deposit and dissemination of scientific research documents, whether they are published or not. The documents may come from teaching and research institutions in France or abroad, or from public or private research centers.
L'archive ouverte pluridisciplinaire HAL, est destinée au dépôt et à la diffusion de documents scientifiques de niveau recherche, publiés ou non, émanant des établissements d'enseignement et de recherche français ou étrangers, des laboratoires publics ou privés. 


\title{
Performance et vieillissement sportif
}

\section{Geoffroy BERTHELOT}

Chercheur en informatique. Institut de Recherche bio-Médicale et d'Épidémiologie du Sport (IRMES), Institut National du Sport, de l'Expertise et de la Performance (INSEP)

Résumé - Depuis l'introduction des Jeux olympiques modernes en 1896, les compétitions sportives ont permis de générer un nombre important de données. Ces dernières, recueillies sur plus de 100 ans, permettent aujourd'hui de définir précisément les contours des capacités physiologiques humaines face à différentes contraintes de temps (courses) ou de distances (lancers, sauts). La technologie, la génétique, la médecine, la géopolitique, les conditions climatiques sont autant de facteurs qui influencent cette performance sportive. Il a été démontré que l'âge chronologique impacte de manière importante le développement de la performance sportive ou intellectuelle. Celui-ci a une forme de $U$ inversé asymétrique, avec des performances aux âges extrêmes qui sont limitées, et un âge optimal de performance aux alentours de 20-30 ans. Cette courbe est également observable dans les performances physiques d'autres espèces comme la souris ou le lévrier. D'autres travaux ont révélé l'aspect bénéfique de l'activité physique à différents moments de la vie, et les gains qu'elle peut procurer en matière de longévité. Il reste cependant beaucoup à faire, notamment pour mieux comprendre comment cette courbe en $U$ est influencée par des paramètres sociétaux comme la consommation d'énergie primaire, l'économie ou l'environnement, par exemple.

Mots clés - performance sportive, vieillissement, limites physiologiques, longévité, durée de vie

\begin{abstract}
Physical performance and ageing
Sporting events havegenerated a significant amount of data since the introduction of the modern Olympics Games in 1896. This data now allows an accurate measurement of human physiological capabilities such as the running speed and jumping, throwing distances. The technological innovations, medicine, geopolitical environment, climate, impact athletic performance. Among those, the chronological age has been shown to significantly affect the development of physical and intellectual performance. The resulting shape is asymmetrical and $U$-inversed, with a similar and limited performance at extreme age bands, and peak performance occurring around the age of 20-30. Other species such as mouse or greyhound also exhibit the same pattern in the age-performance relationship. A few studies have demonstrated the beneficial aspect of physical activity at different age, and the provided gains in terms of longevity. However, further investigations are needed to better understand how the primary energy consumption, economic and environmental aspects can impact this $U$ shaped curve.
\end{abstract}

Keywords - sport performance, ageing, physiological limits, longevity, lifespan 
Quand Pierre de Coubertin fait renaître les Jeux olympiques en 1896, il introduit également la devise olympique officielle : "Citius, Altius, Fortius ». L'objectif était de recréer une compétition internationale, où les athlètes du monde entier se rencontreraient pour repousser les limites physiologiques humaines. Le record du monde symbolise cette lutte ultime et est devenu le symbole de la progression des capacités physiques de l'homme moderne. Après plus de 100 ans de compétition, de nombreux records ont été battus dans de nombreuses disciplines et permettent aujourd'hui d'avoir une idée très précise des capacités humaines sous différentes contraintes. Différentes analyses portant sur le développement historique des performances sportives mettent en évidence deux faits majeurs : la performance physique est limitée (Berthelot et al., 2008, 2010 ; Blest, 1996 ; El Helou, 2011; El Helou et al., 2010 ; Nevill et Whyte, 2005) et dépendante d'un nombre considérable de paramètres. Les hypothèses avancées pour expliquer l'apparente limitation des performances sportives incluent une meilleure implication et structuration des organismes de lutte contre le dopage, un épuisement du vivier mondial (c'est-à-dire un ralentissement dans le développement des processus de détection et de sélection des morphotypes les plus adaptés à une contrainte spécifique : grands - mais pas trop - athlètes africains sur le marathon, etc.), un cadre réglementaire strict limitant les innovations technologiques (combinaison en natation par exemple), etc. Certaines de ces études s'appuient sur un nombre conséquent de données : plus de 3000 records du monde et 40000 performances sportives. La température, l'humidité (El Helou et al., 2012), la génétique (Eynon et al., 2011), la morphologie (Sedeaud et al., 2014), la technologie (Neptune

\section{Figure I - Développement historique des performances sportives.}
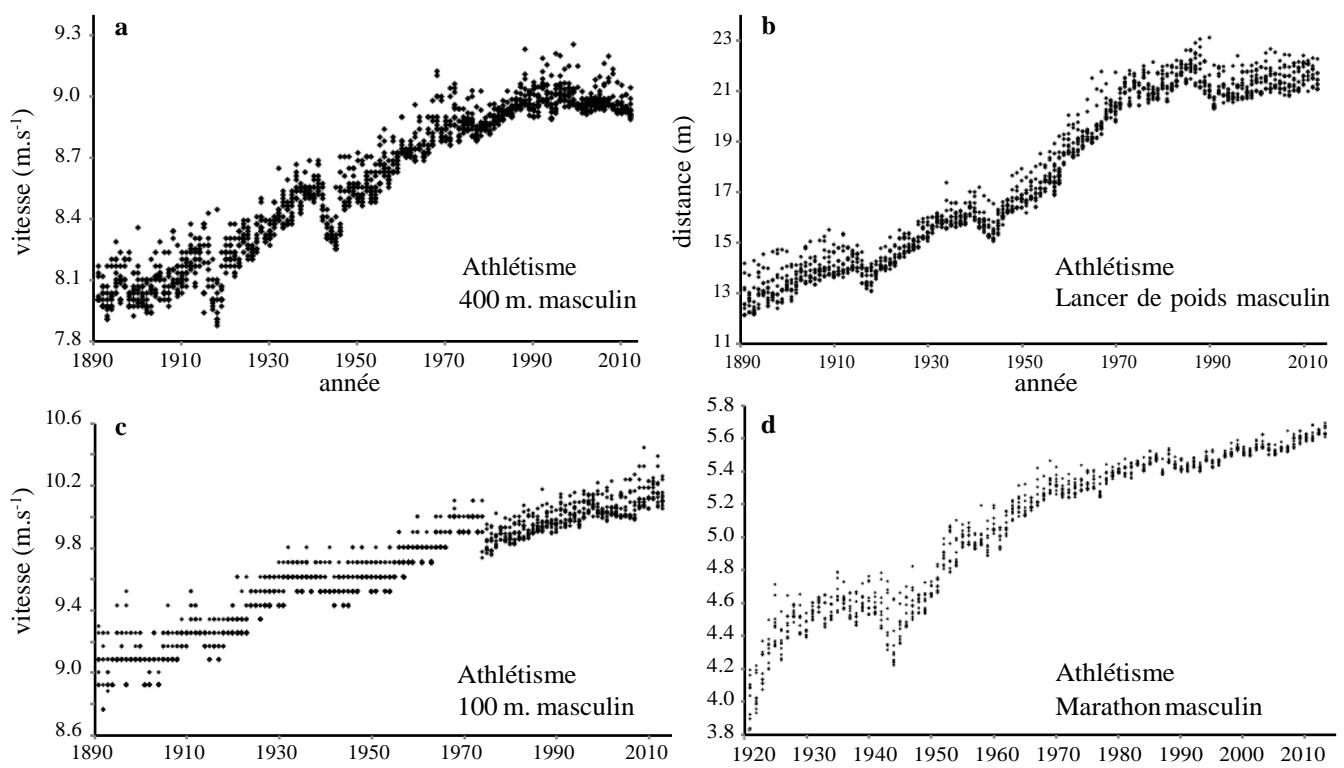

II s'agit des 10 meilleures performances mondiales annuelles de 1896 à 2014 en athlétisme (course de $400 \mathrm{~m}$ (a) et lancer de poids (b)). On constate que ce développement n'est pas linéaire, et admet une limite. Quelques rares épreuves montrent néanmoins une récente progression, comme le $100 \mathrm{~m}$ ou le marathon (panneaux c et d). Le recul historique met toutefois en évidence un développement exponentiel qui tendrait vers une limite. 
et al., 2009), la géopolitique (Guillaume et al., 2009), etc., sont autant de paramètres qui modifient la performance sportive et la rendent si difficilement prévisible.

\section{Développement de la performance en fonction de l'âge}

Parmi cette liste de paramètres, de nombreuses études ont démontré que l'âge est un déterminant majeur de la performance sportive (Baker et al., 2003 ; Tanaka et Seals, 2008). En effet, les paramètres physiologiques sont altérés avec l'âge et il devient de plus en plus difficile pour un athlète de haut niveau de reproduire une performance à mesure que son âge progresse après 30-40 ans. De manière générale, les scientifiques cherchent à caractériser la phase de décroissance de la performance en fonction de l'âge chez des athlètes masters (au-delà de 30 ans). Ces travaux montrent que la performance décroît de manière non linéaire, avec une forme pouvant être approchée par une fonction quadratique ou exponentielle. Seul Dan H. Moore a publié un article analysant le développement de la performance sportive sur la totalité du spectre de l'âge chronologique (Moore, 1975). Cet article, publié dans la revue Nature en 1975, est basé sur l'analyse des performances de quelques populations de sportifs de haut niveau en athlétisme (Moore, 1975). Il a mis en évidence que les performances suivent une phase de croissance jusqu'à l'âge de 20-25 ans, puis une phase de décroissance. Moore a considéré l'enveloppe convexe maximale des performances pour chaque classe d'âge. C'est-à-dire qu'il a sélectionné, pour chaque âge, la meilleure performance observée dans sa population. Plus récemment, d'autres travaux ont montré que cette courbe biphasique est redondante et qu'on la retrouve également dans des populations de nageurs et de tennismen (Guillaume et al., 2011) (Figure 2). Plus surprenant, si l'on considère une performance intellectuelle, on constate que cette courbe se retrouve également chez les grands maîtres d'échecs (Berthelot et al., 2012). Les échecs représentent également une performance intellectuelle où l'anticipation, la spatialisation et la stratégie jouent un rôle important. De nombreux processus cognitifs sont sollicités à cette occasion, et l'on peut dire que les scores aux échecs représentent une appréciation quantitative - moyennant les biais de mesure qui existeront toujours - de la synthèse de certains de ces processus. D'autres auteurs, comme Germine et al., utilisent d'autres méthodes comme la capacité à reconnaissance des visages (le Cambridge Memory Test v2) pour évaluer le développement ou le déclin de certaines de nos capacités cognitives en fonction de l'âge (Germine, Duchaine et Nakayama, 2011). Bien sûr, aucun des exemples mentionnés, y compris les échecs, ne sollicitent complètement (c'est-à-dire de manière exhaustive) l'ensemble des capacités cognitives. Et le bon sens nous dira qu'il existe toute une panoplie de formes d'intelligences : certaines personnes sont plus douées pour les lettres que pour les chiffres par exemple. Toutes ces courbes sont modélisables par une seule et même fonction mathématique, laissant supposer qu'il existerait possiblement un processus biphasique résultant de mécanismes biologiques communs. Bien sûr, il existe quelques différences : les vitesses de croissance et de décroissance de la performance sont sensiblement différentes, les âges où la performance est maximale ne sont pas parfaitement identiques. On note cependant que ces paramètres sont très proches. Par exemple, l'âge où la performance maximale est observée réside dans un intervalle très restreint : autour de 
Figure 2 - Développement des performances en fonction de l'âge pour le $100 \mathrm{~m}$ hommes

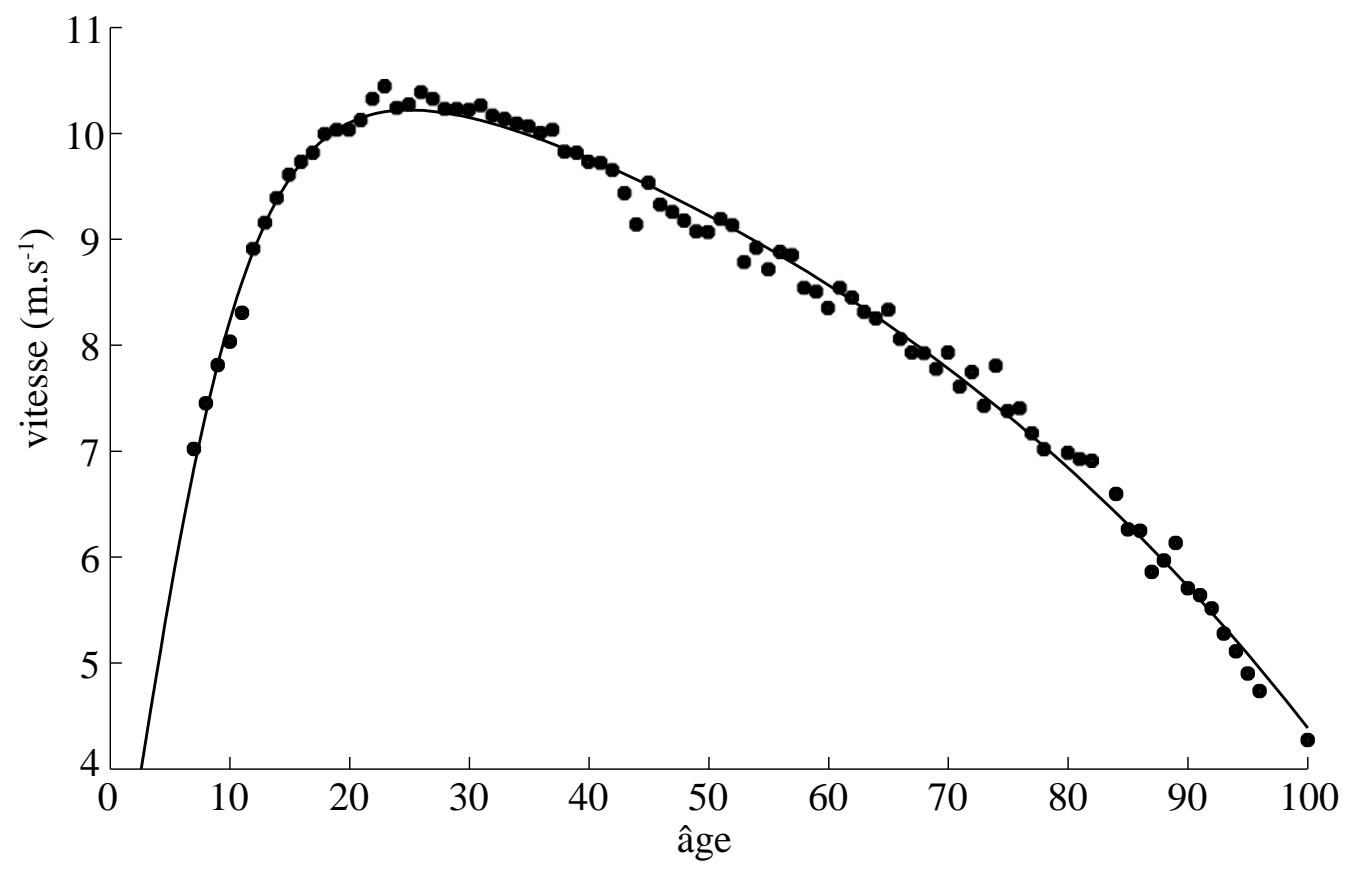

Pour chaque classe d'âge, la meilleure performance observée est retenue (traduite en vitesse). La courbe résultante comporte une phase croissante et une phase décroissante et n'admet qu'un seul pic (comportement unimodal).

20-30 ans. Les performances des joueurs d'échecs semblent décliner plus lentement que les performances des athlètes de haut niveau. Néanmoins, ce processus est toujours unimodal : il n'admet qu'un seul sommet. Il n'existe aucune évidence d'une autre dynamique dans la littérature, qui laisserait supposer qu'on puisse répéter de nombreuses fois une performance tout au long de sa vie.

D'autres auteurs ont démontré que ce processus semble également apparaître chez d'autres espèces que l'homme (abeilles ; Vance et al., 2009) et dans les plantes (Kasemsap et al., 1997) en observant le rendement de la photosynthèse par âge de la feuille dans les champs de coton. Ce processus se retrouve aussi dans d'autres phénomènes biologiques comme la densité minérale osseuse (Boot et al., 2010), le développement de la fonction pulmonaire (Schoenberg et al., 1978), la capacité cognitive (Germine et al., 2011 ; Salthouse, 2009), ou la reproduction humaine (Kühnert et Nieschlag, 2004). Cette relation existe aussi chez les lévriers et les souris : les données de compétition des lévriers sont largement disponibles et il existe par exemple plus de 48000 performances sur des distances de $480 \mathrm{~m}$ documentées de 2002 à 2012 permettant de tester cette assertion. Chez les souris, les données de l'activité libre (nombre de tours de roues) recensées auprès de 224 souris pendant toute la durée de leur vie et comptabilisant plus de 14000 performances (Bronikowski et al., 2006 ; Morgan et al., 2003) montrent que le développement de la performance en fonction de l'âge se retrouve bien chez ces deux espèces. Et ce, malgré un biais de mesure de la performance directe chez la souris. 


\section{Performance et durée de vie}

L'allongement de l'espérance de vie peut aussi être considéré comme une amélioration de la performance du corps humain. Cette capacité s'est développée de manière importante pendant le siècle dernier, de manière similaire aux performances sportives. Elle a bénéficié des nouvelles technologies et d'autres facteurs que l'on retrouve également comme paramètres influents de la performance sportive. Alors que cette dernière admet une limite (Figure 1), l'existence d'une limite de la durée de vie est une question qui divise fortement la communauté scientifique. Certains proposent une progression constante de l'espérance de vie (un estimateur de la durée de vie), alors que d'autres suggèrent qu'elle est limitée. Leonard Hayflick ira même jusqu'à replacer ce questionnement dans le contexte sportif : "To imagine that the current rate of life expectancy increase will continue indefinitely is as absurd as extrapolating the diminishing time taken to run a mile and concluding that it will sooner or later be done in one second " (cité dans Watts, 2011). Une étude récente explore cette question à travers l'analyse de deux cohortes spécifiques (Antero-Jacquemin da Silva et al., 2014) :

- la cohorte des Olympiens français, constituée de plus de 19000 individus (environ 18000 hommes et 1200 femmes), nés entre 1828 et 1991 et ayant participé au moins une fois aux Jeux olympiques;

- la cohorte GRG (Gerontology Research Group) des supercentenaires (Coles, 2008), totalisant quelque 1400 individus à travers le monde (environ 130 hommes et 1300 femmes) ayant plus de 110 ans. Cette cohorte est fiable et vérifiée régulièrement (se reporter au site du GRG) (Coles, 2008).

Bien que ces cohortes soient de petite taille, l'étude met en évidence qu'elles évoluent de façon similaire, et ne sont donc pas complètement indépendantes (Antero-Jacquemin da Silva et al., 2014). Ainsi, les impacts historiques et culturels, comme les guerres mondiales par exemple, sont observables dans ces deux populations. Enfin, l'étude montre également un phénomène de compression des durées de vies élevées, phénomène déjà mis en évidence par Kannisto (2000). Il se traduit d'une part par une réduction importante de la mortalité infantile et d'autre part par une réduction de la mortalité aux âges avancés. Cette augmentation de survie semble possible jusqu'à une certaine limite, car on constate ensuite une brutale augmentation de la mortalité, due à l'affaiblissement inexorable et conjuguée des défenses de l'organisme et de ses capacités de régénération. Ce phénomène est décrit comme une compression de la courbe de mortalité : le nombre de durées de vies élevées augmente jusqu'à un certain point avant de s'effondrer, illustrant un phénomène de rectangularisation. La cohorte GRG montre ainsi une augmentation de la densité de supercentenaires âgés de 110 ans environ, mais la densité de personnes plus âgées reste stable. Le record maximal observé reste à ce jour celui de Jeanne Calment, décédée en 1997 à l'âge de 122 ans. Ces deux cohortes semblent donc montrer que la durée de vie humaine est limitée, même s'il faudra probablement mener des analyses supplémentaires dans d'autres types de populations (population générale notamment) pour pouvoir généraliser cette tendance. Le fait que les records de ces deux paramètres physiologiques (durée de vie et performance sportive) soient limités apparaît cependant cohérent au regard des connaissances établies dans la biologie aujourd'hui (Lui et Baron, 2011). Une autre inconnue est la relation liant qualité de vie et performance. Depuis peu, 
on admet que l'activité physique peut éventuellement se substituer à certaines médications. Un travail récent et portant sur une cohorte de 786 cyclistes ayant participé au moins une fois au Tour de France entre 1947 et 2012, montre que ces athlètes de haut niveau semblent avoir une survie plus importante que la population générale. Les causes globales et spécifiques de mortalité ont été analysées et cette population présente un taux de mortalité de $41 \%$ inférieur à la population générale (Marijon et al., 2013). Certaines inconnues subsistent, notamment en ce qui concerne les nouvelles substances dopantes et leurs implications futures dans la mortalité ou la survenue de pathologies. Toutefois, nous ne possédons pas un recul suffisant aujourd'hui pour pouvoir y répondre.

\section{Expansion historique}

La durée de vie et la performance sportive ont augmenté au cours du XX $\mathrm{X}^{\mathrm{e}}$ siècle. On peut donc supposer que la courbe biphasique de la performance en fonction de l'âge est également impactée par le temps : elle a probablement subi une expansion. Cette dernière a précédemment été décrite comme une expansion phénotypique (Figure 3 et Berthelot et al., 2012). Ainsi, les performances à différents âges pour différentes périodes historiques ont probablement augmenté, grâce à la technologie, la médecine et aux facteurs sociaux. Ces derniers ont permis d'accroître à la fois la durée de vie humaine et la performance sportive vers des valeurs plus élevées. Il est toutefois difficile de quantifier précisément l'augmentation de cette enveloppe, car les données permettant d'établir la relation entre l'âge et la performance sont disparates avant 1980. Nous pouvons toutefois supposer que cette enveloppe est bornée, car il existe des limites physiologiques à la performance sportive et il existe probablement des limites concernant la durée de vie humaine.

Figure 3 - Illustration conceptuelle de l'expansion phénotypique, qui s'est probablement produite depuis la révolution industrielle à nos jours

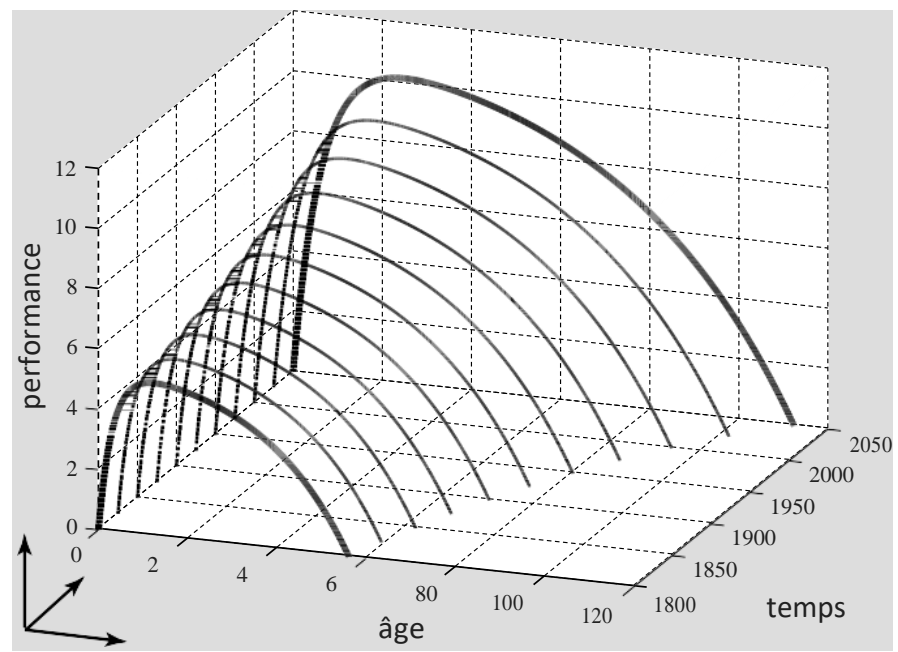

Unités arbitraires : axe $X$ : âge chronologique ; axe $Y$ : performance en vitesse, distance, force, etc. ; axe $Z$ : temps en année. 
Un des déterminants majeurs de cette expansion phénotypique réside dans notre consommation de nourriture et notre utilisation intensive de l'énergie primaire (Fogel, 2004 ; Smith et al., 2013). De nombreux auteurs ont décrit le développement important de cette consommation d'énergie depuis la révolution industrielle. Son utilisation, de plus en plus massive, a permis de libérer du temps horaire pour l'éducation et les loisirs, par exemple, au détriment d'activités autrefois chronophages : récolte de nourriture, production des matériaux, construction des habitats, etc. (Sue, 1994). La production d'énergie a également permis l'essor de nouvelles technologies afin d'explorer l'infiniment petit (accélérateurs de particules), de développer des transports plus rapides et plus fiables, des innovations permettant de battre des records dans le sport (utilisation de la fibre de carbone dans la perche, le vélo, les bateaux, patins clap en patinage, etc.). Dans le domaine sportif, les souffleries ou les bassins d'essais servent aujourd'hui à la conception et au test des combinaisons de natation, de ski, de patinage de vitesse... et à tester l'aérodynamisme des nouveaux matériels : profilage des roues et des guidons, etc. Ces souffleries sont un exemple de matériel énergivore (jusqu'à 100 mégawatts) qui permet de concevoir des produits technologiques toujours plus performants et visant à améliorer la performance. R. Fogel a souligné que des changements technologiques majeurs (pénicilline, électrification, machine de Watt, téléphone, mécanisation de l'agriculture, premiers ordinateurs, etc.) ont conduit à une forte amélioration de l'efficacité des traitements médicaux et de l'accès au soin, une augmentation de notre taille et de notre espérance de vie sur les 200 dernières années. Aujourd'hui, l'agriculture et les procédés alimentaires nécessitent une quantité d'énergie importante : de l'ordre de 9,2 millions de tonnes équivalent pétrole par an (énergies directes et indirectes), soit 5,7 \% de la consommation d'énergie finale (énergie consommée par l'utilisateur) en 2005 (voir G. Bazin et l'Académie d'Agriculture de France travaux du groupe "énergie » réalisés en France en 2007). L'utilisation de cette énergie a permis de faciliter l'accès à la nourriture dans les pays développés. Toutefois, depuis les dernières décennies, l'alimentation et les modes de vie ont évolué : moins d'activité physique quotidienne, apport insuffisant en fruits et légumes (et donc en glucides complexes et fibres), augmentation de la consommation de graisses, de sel et de sucres (Enquête INCA 2 réalisée par l'Afssa). Ce basculement vers un mode de vie sédentaire avec un apport calorique et glycémique excessif est à l'origine d'une forte augmentation de la proportion d'obèses dans plusieurs pays (Malik, Willett et $\mathrm{Hu}, 2013$; Wang et Lobstein, 2006). L'augmentation de la production énergétique a également permis l'essor de nouvelles technologies dans le sport, avec des combinaisons conçues en collaboration avec les ingénieurs de la NASA et faisant appel à des souffleries. Un autre exemple de telle consommation importante d'énergie est l'utilisation de patinoires ou de bassins dits permanents où l'eau est disponible à une température idéale pour l'entraînement tout au long de l'année. On imagine l'énergie dépensée dans les processus de détection des athlètes, dans l'organisation des compétitions et l'acheminement du matériel nécessaire, dans l'entraînement des sportifs (on utilise aujourd'hui des outils technologiques très pointus et coûteux en énergie) avec pour seul objectif l'optimisation de la physiologie humaine. Ce processus est concordant avec l'augmentation progressive du nombre d'athlètes participant à des épreuves physiques dans les catégories masters et plus. Dans un contexte plus général, l'utilisation des technologies et de l'énergie permet à la population générale de vieillir en meilleure santé, pour peu que cette population se trouve dans un pays développé. On soigne aujourd'hui mieux le corps humain, grâce une meilleure connaissance des processus physiologiques impliqués dans les maladies 
et à des médicaments et des thérapies plus performants. De grandes étapes ont été franchies en médecine préventive, en étiopathogénie (l'identification des causes et de facteurs d'une maladie comme le tabagisme, l'amiante, etc.) et dans les outils technologiques de détection et de soin : scanner, IRM, miniaturisation des capteurs embarqués, accélérateurs de particules en médecine, etc. Toutes ces technologies sont de fortes consommatrices d'énergie primaire, mais permettent de maintenir le corps apte à la pratique d'activité physique à haut niveau à des âges plus tardifs. Cela conduit à une plus grande participation et un plus grand investissement dans les épreuves tels que le marathon, l'ultra-marathon ou l'ironman, ou d'excellentes performances sont observées à des âges aux alentours de 30-40 ans. La courbe biphasique (Figure 2) approche donc progressivement d'une limite, mais ne change pas fondamentalement de dynamique et reste unimodale (Figure 3). Certaines innovations technologiques actuellement utilisées chez les sportifs pourraient avoir un impact sur les catégories d'âges plus avancées. C'est déjà le cas avec la démocratisation des outils connectés comme les montres, les bracelets et bientôt le tee-shirt intelligent, qui permettent de mieux suivre son évolution et de personnaliser son entraînement physique. Dans la pratique de l'activité physique comme loisir et dans le sport professionnel, on s'oriente progressivement vers l'utilisation massive des capteurs pour mesurer plus finement les paramètres physiques afin d'adapter un comportement à des objectifs personnels ou d'équipe. Cette technologie pourrait par exemple être utilisée pour réaliser des chambres entièrement connectées afin de lever automatiquement des alertes chez les malades à l'hôpital. Il reste qu'une avalanche d'informations provenant de tels capteurs pose la question du respect de la vie privée. Il existe également de multiples difficultés lors de l'emploi d'un grand nombre de capteurs, comme l'analyse de la quantité d'information fournie en temps réel, par exemple. Mais on converge progressivement vers une résolution d'une partie de ces difficultés.

\section{Conclusion}

Les performances sportives modernes reflètent les mouvements historiques et turbulents de notre société. Les événements majeurs, tels que les guerres, impactent leur développement. La performance est aussi un révélateur des capacités physiologiques humaines. En tant que telle, elle permet de quantifier précisément certaines relations biologiques comme celle liant le développement de la performance physique ou intellectuelle et le vieillissement. Cette dernière a la forme d'un U inversé asymétrique et s'observe chez l'homme ou le lévrier. Elle semble se décliner à différentes échelles : capacité pulmonaire, densité minérale osseuse, etc.

D’un point de vue général, la performance se dégrade après un âge de pic qui apparaît dans la première moitié de la vie, aux alentours de 20-30 ans pour les humains. D'autres études suggèrent que la pratique régulière d'activité physique permet d'améliorer la qualité de vie, voire de limiter certaines pathologies. La performance est donc fortement liée à la condition physique et reste un estimateur de la durée de vie. Toutefois, il reste encore de nombreux paramètres à étudier pour mieux comprendre les mécanismes aboutissant aux phases de progression et de déclin de nos capacités physiques ou intellectuelles. Des travaux sont en cours dans différents laboratoires pour essayer de quantifier la sénescence cellulaire et d'en mesurer l'impact sur la fonctionnalité. Il y a fort à parier qu'une autre courbe en $\mathrm{U}$ inversée se cache dans cette relation. 


\section{RÉFÉRENCES}

Antero-Jacquemin da Silva, J., Berthelot, G., Marck, A., Noirez, P., Latouche, A. et Toussaint, J.-F. (20I4). Learning From Leaders: Life-span Trends in Olympians and Supercentenarians. The Journals of Gerontology Series A: Biological Sciences and Medical Sciences, glu130.

Baker, A. B., Tang, Y. Q. et Turner, M. J. (2003). Percentage decline in masters superathlete track and field performance with aging. Experimental aging research, 29(I), 47-65.

Berthelot, G., Len, S., Hellard, P., Tafflet, M., Guillaume, M., Vollmer, J.-C., ...Toussaint, J.-F. (20I2). Exponential growth combined with exponential decline explains lifetime performance evolution in individual and human species. Age, 34(4), I00I-I009.

Berthelot, G., Tafflet, M., El Helou, N., Len, S., Escolano, S., Guillaume, M., ...Toussaint, J.-F. (20I0). Athlete atypicity on the edge of human achievement: performances stagnate after the last peak, in 1988. PLoS One, 5(I), e8800.

Berthelot, G., Thibault, V., Tafflet, M., Escolano, S., El Helou, N., Jouven, X., ...Toussaint, J.-F. (2008). The citius end: world records progression announces the completion of a brief ultra-physiological quest. PLoS One, $3(2)$, el 552.

Blest, D. C. (1996). Lower bounds for athletic performance. The Statistician, 243-253.

Boot, A. M., de Ridder, M. A., van der Sluis, I. M., van Slobbe, I., Krenning, E. P. et de Muinck Keizer-Schrama, S. M. (2010). Peak bone mineral density, lean body mass and fractures. Bone, 46(2), 336-34I.

Bronikowski, A. M., Morgan, T. J., Garland, T. et Carter, P. A. (2006). The evolution of aging and age-related physical decline in mice selectively bred for high voluntary exercise. Evolution, 60(7), I494-I508.

Coles, L. S. (2008). Validated worldwide supercentenarians, living and recently deceased. Rejuvenation research, 11(I), 269-272.

El Helou, N. (20II). Évolution des performances sportives : apport de l'épidémiologie pour l'analyse des performances sportives et des influences physiologiques, technologiques, génétiques et environnementales sur les progressions humaines au cours de l'ère olympique. Paris 5.

El Helou, N., Berthelot, G., Thibault, V., Tafflet, M., Nassif, H., Campion, F., ...Toussaint, J.-F. (20I0). Tour de France, Giro, Vuelta, and classic European races show a unique progression of road cycling speed in the last 20 years. Journal of sports sciences, 28(7), 789-796.

El Helou, N., Tafflet, M., Berthelot, G., Tolaini, J., Marc, A., Guillaume, M., ...Toussaint, J.-F. (20I2). Impact of environmental parameters on marathon running performance. PLoS One, 7(5), e37407.

Eynon, N., Ruiz, J. R., Oliveira, J., Duarte, J. A., Birk, R. et Lucia, A. (20II). Genes and elite athletes: a roadmap for future research. The Journal of physiology, 589(13), 3063-3070.

Fogel, R. (2004). Technophysio evolution and the measurement of economic growth. Journal of Evolutionary Economics, 14(2), 217-221.

Germine, L. T., Duchaine, B. et Nakayama, K. (20II). Where cognitive development and aging meet: Face learning ability peaks after age 30. Cognition, 118(2), 20I-2I0.

Guillaume, M., El Helou, N., Nassif, H., Berthelot, G., Len, S., Thibault, V., ...Toussaint, J.-F. (2009). Success in developing regions: World Records evolution through a geopolitical prism. PLoS One, 4(I0), e7573.

Guillaume, M., Len, S., Tafflet, M., Quinquis, L., Montalvan, B., Schaal, K., ...Toussaint, J.-F. (20II). Success and decline: top 10 tennis players follow a biphasic course. Medicine and science in sports and exercise, 43(I I), $2148-2154$.

Kannisto, V. (2000). Measuring the compression of mortality. Demographic Research, 3(6), 24.

Kasemsap, P., Crozat, Y. et Satakhun, D. (1997). Cotton leaf photosynthesis and age relationship is influenced by leaf position. Nat Sci, 31, 83-92. 
Kühnert, B. et Nieschlag, E. (2004). Reproductive functions of the ageing male. Human Reproduction Update, 10(4), 327-339.

Lui, J. C. et Baron, J. (20I I). Mechanisms limiting body growth in mammals. Endocrine reviews, 32(3), 422-440.

Malik, V. S., Willett, W. C. et Hu, F. B. (20I3). Global obesity: trends, risk factors and policy implications. Nature Reviews Endocrinology, 9(1), 13-27.

Marijon, E., Tafflet, M., Antero-Jacquemin, J., El Helou, N., Berthelot, G., Celermajer, D. S., ...Empana, J.-P. (2013). Mortality of French participants in the Tour de France (1947-20I2). European heart journal, 34(40), 3।45-3I50.

Moore, D. H. (1975). A study of age group track and field records to relate age and running speed. Nature, 253, 264-265.

Morgan, T. J., Garland, T. et Carter, P. A. (2003). Ontogenies in mice selected for high voluntary wheelrunning activity. I. Mean ontogenies. Evolution, 57(3), 646-657.

Neptune, R. R., McGowan, C. P. et Fiandt, J. M. (2009). The influence of muscle physiology and advanced technology on sports performance. Annual review of biomedical engineering, 11, 8I-I07.

Nevill, A. M. et Whyte, G. (2005). Are there limits to running world records? Medicine and Science in Sports and Exercise, 37(10), 1785-1788.

Salthouse, T. A. (2009). When does age-related cognitive decline begin? Neurobiology of aging, 30(4), 507-5I4.

Schoenberg, J. B., Beck, G. J. et Bouhuys, A. (1978). Growth and decay of pulmonary function in healthy blacks and whites. Respiration physiology, 33(3), 367-393.

Sedeaud, A., Marc, A., Marck, A., Dor, F., Schipman, J., Dorsey, M., ...Toussaint, J.-F. (20I4). BMI, a performance parameter for speed improvement. PloS one, 9(2), e90I83.

Smith, K. R., Frumkin, H., Balakrishnan, K., Butler, C. D., Chafe, Z. A., Fairlie, I., ...McKone, T. E. (20I3). Energy and human health. Annual Review of public health, 34, I59-188.

Sue, R. (1994). Temps et ordre social. Paris, France : Presses universitaires de France.

Tanaka, H. et Seals, D. R. (2008). Endurance exercise performance in Masters athletes: age-associated changes and underlying physiological mechanisms. The Journal of physiology, 586(I), 55-63.

Vance, J. T., Williams, J. B., Elekonich, M. M. et Roberts, S. P. (2009). The effects of age and behavioral development on honey bee (Apis mellifera) flight performance. Journal of Experimental Biology, 212(I6), 2604-26II.

Wang, Y. et Lobstein, T. I. M. (2006). Worldwide trends in childhood overweight and obesity. International Journal of Pediatric Obesity, 1(I), II-25.

Watts, G. (20II). Leonard Hayflick and the limits of ageing. The Lancet, 377(9783), 2075.

email auteur : geoffroy.berthelot@insep.fr 